\title{
OSAHS in adolescents: clinical presentation and differential diagnosis
}

\author{
N. Beydon
}

APHP, Respiratory and Sleep Functional Exploration Unit, Armand Trousseau Hospital, France, INSERM U938, Saint-Antoine Research Center, Paris, France

\begin{abstract}
Adolescent experiment widespread changes as he/ she goes through this transitional period from childhood to adulthood. Sleep is no exception to this changeover with, as consequences, modifications of the clinical pre- sentation of subjects with Obstructive Sleep Apnea- Hypopnea Syndrome (OSAHS) and potential different alternative diagnosis.
\end{abstract}

\section{KEYWORDS}

Obstructive Sleep Apnea-Hypopnea Syndrome (OSAHS), adolescent, diagnostic

The characteristics of obstructive sleep apnea-hypopnea syndrome (OSAHS) in adolescents are poorly known because of the scarcity of research on the same. The most frequently asked questions are related to the risk factors and the clinical and physiological specificities of sleep at this age.
What is the relationship between childhood and adolescent OSAHS? How does the upper respiratory tract (URT) and sleep patterns change during adolescence? What are the specific symptoms of adolescent OSAHS?

\section{WHAT IS THE RELATIONSHIP BETWEEN CHILDHOOD OSAHS AND ADOLESCENT OSAHS}

\section{Simple snorers}

Before examining the relationship between childhood and adolescent OSAHS, it is worthwhile to consider how snoring develops among children, because OSAHS is often accompanied by snoring. In Hong Kong, from a general population of 619 Chinese children aged 6-13 years who underwent polysomnography (PSG), 161 children were identified as simple snorers, (SS) which means that the PSG showed an absence of OSAHS?. Seventy-four of these 161 simple snorers were re-evaluated $4.6 \pm 0.6$ years later and it was found that $30 \%$ of them remained SS, 25\% presented with neither

Address for correspondence:

Nicole Beydon - Hôpital Armand Trousseau

26 Avenue du Docteur Arnold Netter - 75012 Paris

E-mail: nicole.beydon@aphp.fr

This is an Open Access article distributed under the terms of the Creative Commons Attribution License (http://creativecommons.org/licenses/by/4.0), which permits unrestricted use, distribution, and reproduction in any medium, provided the original work is properly cited. 
snoring nor OSAHS, and $35 \%$ had an apnea-hypopnea index (AHI) of $>1 / h$, whereas only $7 \%$ presented with a moderate to severe OSAHS (AHI $\geq 5 / h$ ). These results indicate a good prognosis of SS in children, with a low likelihood of progression to OSAHS in adolescence.

\section{Follow-up of children with or without OSAHS from childhood to adolescence}

Three prospective cohort studies aimed at North American populations among which the majority of subjects were identified as at-risk (premature babies and ethnic minorities, particularly black ethnicity) have recently established the link between childhood and adolescent sleep disorders ${ }^{1,4,12}$. This was achieved by monitoring children with an average age of 4.6-8.4 years old from whom two PSG recordings were taken (the first one in early childhood and the second one during adolescence). The primary findings of these studies are summarized in Table 1. It is interesting to note the differences in frequency of detection, remission, and incidence of OSAHS depending on whether or not mild OSAHS (AHI 1-5/h.) is included in the OSAHS group. Notably, almost all of the instances of moderate to severe childhood OSAHS cases (AHI between 5 and $10 / h$ or $>10 / h$ respectively) had regressed by the time the second recording was done in adolescence.

The second interesting point of these studies is the study of the risk factors for OSAHS in adolescence among these children. The three studies identified statistically significant risk factors such as male sex, being overweight in terms of true obesity, which began either in childhood or adolescence or quite simply due to a change in the body mass index (BMI) trajectory pattern.

In two studies, the clinical signs (including snoring) in childhood did not constitute a risk factor of OSAHS in adolescence ${ }^{4,12}$. In the Spilsbury et al. ${ }^{12}$ study, a medical history of adenoidectomy or tonsillectomy performed before the first recording $(n=35)$ or between the two recordings $(n=24)$ was a risk factor for the development of OSAHS in adolescence even after BMI adjustments were made. Goodwin et al. ${ }^{4}$ focused specifically on the effect of having a tonsillectomy between the two recordings. Among these children who underwent the procedure between the two PSGs, adolescents had a higher incidence of OSAHS (defined as a low $\mathrm{AHI}$ threshold $\geq 1$ ) than the younger children in the population (30\% versus 10\%, respectively) and their rate of OSAHS remission was also lower. In summary, ENT surgical intervention appears to be a predisposing factor for adolescent OSAHS, thereby supporting the hypothesis that children who are operated on for adenoid or tonsillar hypertrophy because of clinical symptoms of OSAHS would have other risk factors for OSAHS. These clinical symptoms begin from childhood, highlighted by tonsillar hypertrophy, which is almost physiological in young children, or appearing after the operation (constant weight gain after tonsillectomy)..

The influence of the subjects' ethnicities was also evaluated in these studies because of the heterogeneity of the ethnic groups studied and their frequency in relation to the total population. However, a study with the majority of black subjects $(36.5 \%$ of the entire population 
studied), who are conventionally considered to be predisposed to develop OSAHS, revealed that belonging to this ethnic group constitutes a risk factor for the development of OSAHS in adolescence (after BMI adjustments) ${ }^{12}$.

It is evident that the risk factors for adolescent OSAHS are quite similar to those for adult subjects-i.e., males and overweight/obese individualswhich are not the same risk factors for childhood OSAHS. Black ethnicity also appears to affect all ages, whereas having an ENT surgical history is rarely reported as a predisposing factor in adults. Therefore, it is conceivable that physiological factors underlie this change in risk factors for OSAHS.

\section{PHYSIOLOGICAL MATURATION OF URT AND SLEEP PATTERNS}

\section{URT physiological evolution}

The occurrence of OSAHS involves abnormalities of the two mechanisms that are responsible for the patency of URT during sleep. These mechanisms are (i) the anatomical component including compliance or collapsibility of URT; (ii) the neurogenic component of the ventilatory control that stimulates the pharyngeal respiratory muscles. It is a known fact that in healthy adults, to prevent the collapse of URT during nonREM sleep, an average negative nasal pressure of $-13.3 \pm 3.2 \mathrm{~cm} \mathrm{H}_{2} \mathrm{O}^{10}$ is required, whereas in adults with OSAHS an average positive nasal pressure of $\left(+3.3 \pm 3.3 \mathrm{~cm} \mathrm{H}_{2} \mathrm{O}\right)$ is sufficient ${ }^{11}$. This difference is due to the elevated

Table I: The follow-up of three cohorts from childhood to adolescence ${ }^{1,4,12}$

\begin{tabular}{|c|c|c|c|c|}
\hline & Initial & Follow-up & Remission & Incidence \\
\hline $\begin{array}{l}\text { Recordings }(n)^{1,4,12} \\
\text { Children } \\
\text { Adolescents }\end{array}$ & 700/503/907 & 319/319/490 & & \\
\hline $\begin{array}{l}\text { Average delay between the } \\
2 \text { recordings } \\
1,4,12\end{array}$ & & 8.4/4.6/8.2 years & & \\
\hline Average age (range) & $8.5-9.5(6-12)$ years & $\begin{array}{l}13.7-17.7 \\
(10-23) \text { years }\end{array}$ & & \\
\hline Obesity & $12 \%-15 \%$ & $15 \%-19 \%$ & & \\
\hline SS & $15 \%-21 \%$ & $9 \%-25 \%$ & $31 \%-60 \%$ & $4 \%-25 \%$ \\
\hline Sleepiness* & $16 \%$ & $15 \%$ & $64 \%$ & $12 \%$ \\
\hline Apneas observed* & $5 \%$ & $2 \%$ & $84 \%$ & $1 \%$ \\
\hline Insomnia* & $30 \%$ & $25 \%$ & $48 \%$ & $17 \%$ \\
\hline $\mathrm{AHI} \geq 1 / \mathrm{h}$ & $11 \%-24 \%$ & $15 \%-30 \%$ & $52 \%-70 \%$ & $10 \%-27 \%$ \\
\hline $\mathrm{AHI} \geq 5 / h$ & $1 \%-5 \%$ & $4 \%-22 \%$ & $91 \%-100 \%$ & $4 \%-11 \%$ \\
\hline $\begin{array}{l}\text { Passage from SS to OSAHS with AHI } \\
\geq 1 / \mathrm{h} \text {. }=26 \% \text {, to } \mathrm{AHI} \geq 5 / \mathrm{h} .=12 \%\end{array}$ & & & & \\
\hline
\end{tabular}


compliance (or increased collapsibility) of URT in adults with OSAHS and translates to a more significant transrespiratory pressure gradient in adults with OSAHS. In healthy children aged $11 \pm$ $3(\leq 16)$ years, it has been shown that the slope of the straight line of nasal transrespiratory pressure-air flow relationship during sleep was much lower than that of healthy adults, in favor of a decreased physiological collapsibility of URT, which is most likely attributable to the relationship between ventilatory control and respiratory compliance ${ }^{8}$.

What about the collapsibility of URT in adolescents? Few studies have been conducted, and there is a lack of data regarding thin children. One study conducted with 27 adolescents, among whom seven had OSAHS, showed a slope of relationship between transrespiratory pressure-air flow during sleep that was higher in adolescents with OSAHS, which provides arguments for a mixed mechanism-anatomical and neurogenic ${ }^{3}$.

However, it was found that adolescents with OSAHS had a significantly higher BMI than control subjects (median [range]: 2.5 [1.7-3.0] versus $0.7[-3.8-2.7]$ z scores respectively; $p<0.001)$. Thus, it is difficult to pinpoint what contributes to the physiological maturation of the possible consequences of excess weight or obesity. However, with regards to the neurogenic aspect (ventilation control), obese adolescents with OSAHS experienced a hypercapnic ventilatory response with a similar level of arousal as obese and thin adolescents who do not have OSAHS, though their ventilatory response was lower during sleep. This finding thereby indicates an inconsistent effect of obesity on neurogenic control $^{16}$. Nonetheless, the effect of weight on the relationship between ventilatory control and OSAHS cannot be deduced because normal-weight adolescents with OSAHS were not included in the study.

\section{Changing sleep patterns in adolescence}

It is a well-known fact that physiological sleep changes with age, both in its structure and in its overall duration and rhythms. Regarding the structure, during adolescence, there is a slight decrease in slow-wave sleep with a concomitant increase in light sleep, while the durations for REM sleep remain stable.

The occurrence of obstructive respiratory episodes is rare in healthy children or adolescents, and no link has been found between $\mathrm{AHI}$ (equal to median [range] $0(0-0.9) / h$.) and the age or the pubertal development in 68 healthy, nonobese children or adolescents (8-18 years; on average \pm SD $13 \pm 3$ years) ${ }^{13}$. It can be surmised that there is no link because obstructive events are rare in healthy subjects. There is reason to believe that if there were subclinical periods of flow limitation in healthy children, they would more frequently lead to microarousal and/or desaturation in the healthy adolescent (they would then develop an $\mathrm{AHI}$, which would increase with age) because of the following: (i) the physiological increase in the number of microarousals ${ }^{2}$ and the decrease in the cortical microarousal threshold during obstructive respiratory events between childhood and

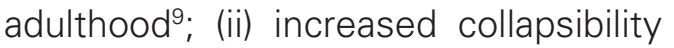
of URT in adolescents (which is similar to that of adulthood), increasing the 
airflow limitation for the same respiratory effort (even nasal pressure) with possible desaturation.

In addition to the stabilization of $\mathrm{AHI}$, changes in circadian rhythm are significant in adolescence and are often the cause of a delayed sleep phase syndrome with delayed physiological drowsiness (delayed melatonin secretion) and late spontaneous awakening (the necessary sleep time not being that different from that of an older child) ${ }^{5}$. These modifications are increased by the increasingly prolonged exposure to light sources in the evenings. Circadian modifications are more severe if bluelight (from screens) exposure is high, which has led to the availability of software/apps allowing the automatic activation of blue-ray filters according to the time of day.

Moreover, this delayed sleep phase syndrome is not taken into account

\section{CLINICAL SIGNS OF ADOLESCENT OSAHS}

First of all, the presence of the previously explained risk factors for OSAHS will more likely result in its diagnosis when dealing with an overweight or obese boy, especially if the weight gain took place after the child attained primary school age. Once again, because of the paucity of information published about adolescents, it is difficult to get an exact idea of the frequency and profile of thin adolescents with OSAHS.

In a cross-sectional study in which relationship between BMI and OSAHS was studied in Caucasian children aged 2-18 years, it was necessary to increase the inclusion period to obtain a greater number of adolescents $(\geq 12$ years) because this population does not frequent sleep centers very often ${ }^{6}$. by school rhythms, because schools do not start classes later to cater to adolescents. Owing to delayed sleep phase syndrome, adolescents create a sleep deficit during the week for which they can only partially compensate on weekends. As a result, there is often a chronic sleep deficit in adolescents. In adults, chronic sleep deprivation is an aggravating factor of OSAHS and promotes weight gain. The influence of adolescent sleep deficit on the onset of OSAHS is poorly understood.

All these data can guide the clinician, confronted with an adolescent or their family complaining about the subject's sleep patterns, to distinguish between those symptoms which are likely to lead to OSAHS and those related to physiological sleep as regards the adolescent's specific lifestyle at that age.

In the final analysis, out of 234 children recorded $(16 \%), 38$ adolescents were included, $34.2 \%$ of whom were thin, $13.2 \%$ were overweight, and $53.6 \%$ were obese. BMI seems to be a risk factor in adolescents, in whom OSAHS risk is increased 3.5 times for each standard deviation (+1 z-score) in excess of the normal BMI range ${ }^{6}$. Caucasian subjects were exclusively included in this study to avoid the known influence of ethnicity.

Let us now discuss the symptoms most frequently found in obese OSAHS adolescents. A study with a restricted sleep recording (only a nasal cannula + pulse oximetry to facilitate apnea scoring) of obese population of adolescents, distinguished three 
groups according to the frequency of recorded apneas: no OSAHS ( $n=24)$, mild OSAHS (apnea index (Al) between $2-5 / h ; n=29$ ), and moderate to severe OSAHS (AI $\geq 5 / h ; n=8)^{14}$. The following symptoms increased in frequency as per Al: snoring, shortness of breath while sleeping, cessations in breathing (apnea) noticed by loved ones, bed-wetting, daytime fatigue, and behavior disorders. The following signs were not statistically different among the three groups: restless sleep, the Epworth Sleepiness Scale scores, morning headaches, learning and concentration disorders, sleepwalking, nightmares, tonsillectomy, mouth breathing, allergies, asthma, smoking, and gastroesophageal reflux disease (GERD) ${ }^{14}$. Another study conducted on a larger sample, using more sensitive neurobehavioral testing and a complete PSG recording, compared 38 obese adolescents with OSAHS (AHI $\geq 5 / h$ ) to 21 obese patients without OSAHS (AHI $<1.5 / h)$ and 36 thin adolescents SS (without OSAHS) ${ }^{15}$. The parental assessment showed more cognitivebehavioral disorders (executive functions, attention, behavioral disorders) in the obese group with OSAHS than in the other two groups, and the adolescents' responses to different questionnaires made it possible to detect more instances of depression and sleepiness (but only in black subjects). Adolescence represents an age when brain development is still in progress. Thus, the impact of these disorders on the subsequent behavior of the adult subject is a question posed by the authors of the study, who advocate treating the adolescent's OSAHS so as to prevent unfavorable neurobehavioral changes ${ }^{15}$.
In summary, according to our current knowledge, adolescents with OSAHS are more likely to be obese boys or adolescents who have gained too much weight in recent years, snorers (but not necessarily from childhood) with possible breathing difficulties or reported nocturnal apnea. Affected adolescents also present with symptoms of depression, impaired executive functioning and social skills, or even attention disorders. The problem is differentiating adolescents with OSAHS from those who are brought in for consultation by their families or schools because they present with similar symptoms such as: daytime sleepiness, declining academic performance, relationship disorders (some tend to be reclusive while others engage in risky behavior), in addition to possible drug or alcohol use that can affect sleep. Among these adolescents, other causes which may explain these neurobehavioral disorders should be sought.

First, delayed sleep phase syndrome which can lead to fatigue even daytime sleepiness and can be responsible for both concentration issues as well as neurobehavioral disorders. In addition, lack of sleep leads to dietary disruptions that promote weight gain (increasing the quantity of snacks as well as one's appetite for sweet and fatty foods). As previously mentioned, weight gain is often considered as an indicator for OSAHS. This phase shift will be highlighted during history taking (sleep and waking hours during the holiday period) or by having the adolescents maintain a sleep diary or wear an actimeter for a few weeks to objectively test it.

Second, it is necessary to eliminate habits that decrease sleep time, such as the use of screens in the evening, 
as they have an excitatory effect due to their content; in addition, as mentioned above, they cause a delay effect on melatonin secretion because of blue-light exposure. In young individuals indulging in online gaming, it may be the case that they reschedule wakeful times in the night to be able to participate in game play. These behaviors are the direct result of recent societal changes and can of course be associated with a delayed sleep phase syndrome and perhaps even true OSAHS.

\section{CONCLUSION}

Adolescent OSAHS is poorly studied and almost exclusively described in obese, male adolescents. However, neither excess weight nor the individual's sex are risk factors among younger children. These changes in risk factors reflect the anatomical maturation of URT and of their neurogenic control. Adolescents with OSAHS will most likely have nocturnal respiratory symptoms whose neurobehavioral consequences are sometimes the only ones known and can easily be blamed for changes inherent in this age group. Therefore, clinicians must be cognizant of how to make such a diagnosis, which can only be confirmed by a PSG recording.

\section{BIBLIOGRAPHY}

1. Bixler EO, et al. Natural history of sleep disordered breathing in prepubertal children transitioning to adolescence. Eur Respir J 2016;47(5):1402-1409.

2. Bonnet MH, Arand DL. EEG arousal norms by age. J Clin Sleep Med 2007;3(3):271-274.

3. Carrera HL, et al. Negative Expiratory Pressure Technique: An Awake Test to Measure Upper Airway Collapsibility in Adolescents. Sleep 2015;38:1783-1791.

4. Goodwin JL, Vasquez MM, Silva GE, Quan SF. Incidence and remission of sleep-disordered breathing and related symptoms in 6- to 17-year old children--the Tucson Children's Assessment of Sleep Apnea Study. J Pediatr 2010;157(1):57-61.

5. Hagenauer MH, Perryman JI, Lee TM, Carskadon MA. Adolescent changes in the homeostatic and circadian regulation of sleep. Dev Neurosci 2009;31(4):276-284.

6. Kohler MJ, et al. Differences in the association between obesity and obstructive sleep apnea among children and adolescents. J Clin Sleep Med 2009;5(6):506-511.

7. Li AM, ZhuY, Au CT, Lee DLY, Ho C, Wing YK. Natural history of primary snoring in schoolaged children: a 4-year follow-up study. Chest 2013;143(3):729-735.

8. Marcus CL, Lutz J, Hamer A, Smith PL, Schwartz A. Developmental changes in response to subatmospheric pressure loading of the upper airway. J Appl Physiol 1999;87(2):626-633.

9. McNamara F, Issa FG, Sullivan CE. Arousal pattern following central and obstructive breathing abnormalities in infants and children. J Appl Physiol 1996;81:2651-2657.

10. Schwartz AR, Smith PL, Wise RA, Gold AR, Permutt S. Induction of upper airway occlusion in sleeping individuals with subatmospheric nasal pressure. J Appl Physiol 1988;64(2): 535-542.

11. Smith PL, Wise RA, Gold AR, Schwartz AR, Permutt S. Upper airway pressure-flow relationships in obstructive sleep apnea. J Appl Physiol 1988;64(2):789-795. 
12. Spilsbury JC, Storfer-Isser A, Rosen CL, Redline S. Remission and incidence of obstructive sleep apnea from middle childhood to late adolescence. Sleep 2015;38(1):23-29.

13. Tapia IE, et al. Polysomnographic values in children undergoing puberty: pediatric vs. adult respiratory rules in adolescents. Sleep 2008;31:1737-1744.

14. Verhulst SL, Franckx H, Van Gaal L, De Backer W, Desager K. The effect of weight loss on sleep-disordered breathing in obese teenagers. Obesity 2009;17(6):1178-1183.

15. Xanthopoulos MS, Gallagher PR, Berkowitz RI, Radcliffe J, Bradford R, Marcus CL. Neurobehavioral functioning in adolescents with and without obesity and obstructive sleep apnea. Sleep 2015;38(3):401-410.

16. Yuan $\mathrm{H}$, et al. Ventilatory responses to hypercapnia during wakefulness and sleep in obese adolescents with and without obstructive sleep apnea syndrome. Sleep 2012;35:1257-1267. 